\title{
Significance of Preoperative Systemic Immune Score for Stage I Gastric Cancer Patients
}

\author{
Jun Lu, ${ }^{1,2,3,4}$ Long-long Cao, ${ }^{1,2,3,4}$ Ping Li, ${ }^{1,2,3,4}$ Jian-wei Xie, ${ }^{1,2,3,4}$ Jia-bin Wang, ${ }^{1,2,3,4}$ \\ Jian-xian Lin, ${ }^{1,2,3,4}$ Qi-yue Chen, ${ }^{1,2,3,4}$ Mi Lin, ${ }^{1,2,3,4}$ Ru-hong Tu, ${ }^{1,2,3,4}$ \\ Chang-ming Huang $\mathbb{D}^{1,2,3,4}$ and Chao-hui Zheng $\mathbb{D}^{1,2,3,4}$ \\ ${ }^{1}$ Department of Gastric Surgery, Fujian Medical University Union Hospital, Fuzhou, China \\ ${ }^{2}$ Department of General Surgery, Fujian Medical University Union Hospital, Fuzhou, China \\ ${ }^{3}$ Key Laboratory of Ministry of Education of Gastrointestinal Cancer, Fujian Medical University, Fuzhou, China \\ ${ }^{4}$ Fujian Key Laboratory of Tumor Microbiology, Fujian Medical University, Fuzhou, China
}

Correspondence should be addressed to Chao-hui Zheng; wwkzch@163.com

Received 13 January 2018; Revised 2 May 2018; Accepted 10 May 2018; Published 11 July 2018

Academic Editor: Fausto Rosa

Copyright (c) 2018 Jun Lu et al. This is an open access article distributed under the Creative Commons Attribution License, which permits unrestricted use, distribution, and reproduction in any medium, provided the original work is properly cited.

\begin{abstract}
Background. Determining preferences regarding the benefits of adjuvant chemotherapy (AC) for stage I GC is critical. Methods. We retrospectively reviewed 1069 patients with pathologically confirmed stage I GC who underwent R0 gastrectomy between 2006 and 2014. Univariate and multivariate survival analyses were conducted. Systemic inflammation factors were used to develop a scoring system for predicting AC benefits. Results. With a median follow-up of 47 months (range 3-113 months), the 5-year overall survival (OS) rate was $90.5 \%$. The patient score was 1 for either a pretreatment hypoalbuminemia or elevated derived neutrophillymphocyte ratio (dNLR) and was 0 otherwise. The SIS served as an independent prognostic factor for reduced OS. AC was delivered to $13.5 \%(144 / 1069)$ of all patients. Compared to surgery alone, AC had no significant effect on survival in both the entire cohort and the IA/IB subgroup. However, in the high-risk group (SIS =2), patients with AC had a significantly better OS than those undergoing surgery alone. Conclusions. Patients with SIS $=2$ may benefit from AC and thus may be considered candidates for adjuvant treatment. However, to confirm our findings, future prospective studies are warranted.
\end{abstract}

\section{Introduction}

Gastric cancer (GC) is the fourth most common cancer and the second leading cause of cancer death worldwide [1]. Unlike the situation for stage II and stage III GC, there is no global agreement on adjuvant chemotherapy (AC) for stage I GC due to (1) the prognosis of stage I GC is relatively good, with over $90 \%$ of 5 -year overall survival [2], (2) although the benefit of AC was established for patients with stage II and stage III GC, clinical trials included stage I population failed to show the benefit of AC over surgery alone [2-7]. However, relapse occurs in a small but definite number of patients, even those with stage I GC, after curative resection [8]. Moreover, recent studies showed that the prognosis and response to chemotherapy is different according to the molecular characteristics of gastric cancer [9-11]. Therefore, it is necessary to identify patients with stage I GC who might receive a therapeutic benefit from $\mathrm{AC}$.

The role of the immune system in cancer has become increasingly prominent [12-17]. In addition to local inflammatory symptoms, cancer patients frequently present with systemic inflammation responses, including increased peripheral blood cell amounts and decreased serum albumin levels [18]. More recently, a study at the Memorial Sloan Kettering Cancer Center suggested that pretreatment neutrophil lymphocyte ratio (NLR) can be used to identify nonmetastatic melanoma patients who are more likely to benefit from adjuvant treatment [19]. In 2012, Proctor et al. [20] implemented a simplified index that is easier to apply to clinical data named the derived neutrophil-lymphocyte ratio (dNLR), and they were also able to demonstrate that the preoperative dNLR had similar prognostic value as the classical NLR. 
TABle 1: Demographic and clinical features.

\begin{tabular}{|c|c|c|c|c|c|}
\hline Characteristics & Number & $\%$ & Characteristics & Number & $\%$ \\
\hline Age, years (median, IQR) & $60(18-93)$ & & ASA class & & \\
\hline Sex & & & $1-2$ & 1020 & 95.4 \\
\hline Male & 795 & 74.4 & $\geq 3$ & 49 & 4.6 \\
\hline Female & 274 & 25.6 & Type of surgery & & \\
\hline CEA level & & & Total gastrectomy & 284 & 26.6 \\
\hline Normal & 871 & 81.5 & Subtotal gastrectomy & 785 & 73.4 \\
\hline Above normal & 116 & 10.9 & Type of LN dissection & & \\
\hline Unknown & 82 & 7.7 & $\mathrm{D} 1$ & 11 & 1.0 \\
\hline CA 19-9 level & & & $\mathrm{D} 1+$ & 549 & 51.3 \\
\hline Normal & 826 & 77.3 & D2 & 509 & 47.7 \\
\hline Above normal & 152 & 14.2 & Lymphovascular invasion & & \\
\hline Unknown & 91 & 8.5 & No & 945 & 88.4 \\
\hline Differentiation & & & Yes & 93 & 8.6 \\
\hline Well or moderate & 603 & 56.4 & Unknown & 31 & 2.9 \\
\hline Poor & 466 & 43.6 & T stage & & \\
\hline Tumor size, $\mathrm{cm}$ & & & $\mathrm{T} 1$ & 821 & 76.8 \\
\hline$<3$ & 590 & 55.2 & $\mathrm{~T} 2$ & 248 & 23.3 \\
\hline$\geq 3$ & 479 & 44.8 & $\mathrm{~N}$ stage & & \\
\hline Tumor location & & & No & 895 & 83.7 \\
\hline Lower & 595 & 55.7 & N1 & 174 & 16.3 \\
\hline Middle & 138 & 12.9 & TNM stage & & \\
\hline Upper & 207 & 19.4 & IA & 635 & 59.4 \\
\hline Multiple & 129 & 12.0 & IB & 434 & 40.6 \\
\hline Haemoglobin, g/l (median, IQR) & $135(53-174)$ & & $\mathrm{AC}$ & & \\
\hline Albumin, g/l (median, IQR) & $40(20-52)$ & & No (surgery alone) & 925 & 86.5 \\
\hline WBC, $10^{9} / 1$ (median, IQR) & $6.0(2.7-16.6)$ & & Yes (surgery $+\mathrm{AC})$ & 144 & 13.5 \\
\hline Neutrophils, $10^{9} / 1$ (median, IQR) & $3.5(0.7-14.6)$ & & Pretreatment hypoalbuminemia & & \\
\hline Platelets, $10^{9} / 1$ (median, IQR) & $217(80-523)$ & & No & 895 & 83.7 \\
\hline $\mathrm{dNLR}$ & & & Yes & 174 & 16.3 \\
\hline$<2$ & 797 & 74.6 & & & \\
\hline$\geq 2$ & 272 & 25.4 & & & \\
\hline
\end{tabular}

$\mathrm{IQR}=$ interquartile range; $\mathrm{AC}=$ adjuvant chemotherapy; $\mathrm{dNLR}=$ neutrophil count to (white cell count minus neutrophil count); $\mathrm{ASA}=\mathrm{American}$ Society of Anesthesiologists. Pretreatment hypoalbuminemia: pretreatment serum albumin $<35 \mathrm{~g} / \mathrm{l}$.

Preoperative serum albumin levels are also reported as prognostic indicators for the prognosis of cancer patients $[21,22]$. These markers are inexpensive to test and routinely performed in clinical setting and hence potentially provide readily available and objective information to help clinicians to estimate patient outcome.

To the best of our knowledge, the potential influence of the pretreatment dNLR and hypoalbuminemia has never been explored in large cohorts of stage I GC patient yet. The aim of the present study was to verify the hypothesis that the systemic immune score (SIS) based on preoperative dNLR and serum albumin is associated with worse survival in diagnosed stage I GC and more importantly to investigate whether SIS can distinguish subgroups of patients who would benefit from AC. If so, such commonly measured SIS could be used in developing a strategy for selecting stage I GC treatments.

\section{Materials and Methods}

2.1. Patients. A total of 1069 patients undergoing R0 resection for stage I GC at Fujian Medical University Union Hospital (FMUUH) from December 2006 to December 2016 were identified from a prospectively maintained database. The following exclusion criteria were then applied: (1) prior gastrectomy, (2) receiving neoadjuvant chemotherapy, (3) noncurative (R1/2) resection, (4) the presence of synchronous malignant disease, (5) autoimmune disorders and recent steroid therapy, and (6) incomplete medical records. Curative resection refers to $\mathrm{R} 0$ resection in Japanese Gastric Cancer Treatment Guidelines 2010 (version 3) [23]. Tumor stages were assessed according to the American Joint Committee on Cancer (AJCC) classification system, 7th edition [24]. Additional demographic and clinical data are 
summarized in Table 1 . The study protocol was approved by the ethical committee of the FMUUH.

2.2. Adjuvant Chemotherapy. Since there is no established treatment strategy for stage I gastric cancer, decisions to administer AC in those patients were based on their surgeons' or oncologists' preference $[25,26]$. According to the treatment protocol of our institute, stage I GC patients with lymph node metastasis, lymphovascular invasion, or other high risks $[27,28]$ are recommended for AC, unless contraindicated by a patient's condition or their refusal. All AC treatments were performed after individually obtaining written informed consent in accordance with the gastric cancer treatment program at the FMUUH that had been approved by its institutional review board. Finally, $144(13.5 \%)$ patients with written informed consent received 5-fluorouracil plus cisplatin AC, at least one cycle [29]. The clinicopathological variables of both groups are summarized in Supplementary Table 1.

2.3. Systemic Immune Markers. Routine clinical laboratory analyses of peripheral blood samples collected within 1 week before the operation were used to ascertain white cell counts, neutrophil counts, and serum albumin. The pretreatment dNLR was calculated as follows: $\mathrm{dNLR}=$ neutrophil count to (white cell count-neutrophil count). The cutoff value (2.0) for the pretreatment dNLR was selected as described previously [20]. The cutoff value for the pretreatment hypoalbuminemia $(35 \mathrm{~g} / \mathrm{l})$ was according to the normal value measured by the used test $[22,30]$. All the measurements were performed in the central biochemical laboratory at the Department of Clinical Biochemistry and Hematology, FMUUH.

We combined the two risks to establish the SIS defined as follows: patients with both elevated dNLR and hypoalbuminemia ( $\mathrm{dNLR} \geq 2$ and albumin $<35 \mathrm{~g} / \mathrm{l}$, resp.) were assigned score 2 , patients with either elevated dNLR or hypoalbuminemia were assigned score 1 , and patients with both decreased $\mathrm{dNLR}$ and normal albumin ( $\mathrm{dNLR}<2$ and albumin $\geq 35 \mathrm{~g} / \mathrm{l}$, resp.) were assigned score 0 .

2.4. Follow-up. All patients were monitored postoperatively by physical examination and laboratory tests, including those for tumor markers (such as CEA and CA 19-9), every 3 months for the first 2 years, every 6 months for the next 3 years, and annually thereafter. In addition, examinations, including chest radiography, abdominopelvic computed tomography (CT), and endoscopy, were performed at least once a year. If necessary, further evaluation, such as positron emission tomography or magnetic resonance imaging, was initiated.

2.5. Statistical Analysis. Overall survival (OS) was defined as the time from the day of surgery to the death from any cause. Univariate survival trends were compared using KaplanMeier curves and significant differences determined via the log rank test, and those variables that achieved statistical significance in the univariate analysis were entered into the multivariable analysis. Multivariate analysis of prognostic factors was conducted with Cox's proportional hazards
TABle 2: Patient baseline characteristics (adjuvant chemotherapy with surgery versus surgery alone).

\begin{tabular}{|c|c|c|c|}
\hline Characteristics & $\begin{array}{l}\text { AC group } \\
(n=144)\end{array}$ & $\begin{array}{l}\text { Non-AC group } \\
\quad(n=925)\end{array}$ & $p$ value \\
\hline Age, years (median, IQR) & $58(18-81)$ & $60(20-93)$ & 0.402 \\
\hline Sex & & & 0.474 \\
\hline Male & 103 & 692 & \\
\hline Female & 41 & 233 & \\
\hline CEA level & & & 0.146 \\
\hline Normal & 112 & 759 & \\
\hline Above normal & 21 & 95 & \\
\hline Unknown & 11 & 71 & \\
\hline CA 19-9 level & & & 0.701 \\
\hline Normal & 111 & 715 & \\
\hline Above normal & 22 & 130 & \\
\hline Unknown & 11 & 80 & \\
\hline \multicolumn{4}{|l|}{ Differentiation } \\
\hline Well or moderate & 78 & 525 & \\
\hline Poor & 66 & 400 & \\
\hline Tumor size, $\mathrm{cm}$ & & & 0.419 \\
\hline$<3$ & 84 & 506 & \\
\hline$\geq 3$ & 60 & 419 & \\
\hline Tumor location & & & 0.591 \\
\hline Lower & 76 & 519 & \\
\hline Middle & 20 & 118 & \\
\hline Upper & 31 & 176 & \\
\hline Multiple & 17 & 112 & \\
\hline Type of surgery & & & 0.522 \\
\hline Total gastrectomy & 43 & 241 & \\
\hline Subtotal gastrectomy & 101 & 684 & \\
\hline TNM stage & & & $<0.001$ \\
\hline IA & 48 & 587 & \\
\hline IB & 96 & 338 & \\
\hline Pretreatment dNLR & & & 0.064 \\
\hline$<2$ & 116 & 681 & \\
\hline$\geq 2$ & 28 & 244 & \\
\hline $\begin{array}{l}\text { Pretreatment } \\
\text { hypoalbuminemia }\end{array}$ & & & 0.260 \\
\hline No & 121 & 774 & \\
\hline Yes & 23 & 151 & \\
\hline
\end{tabular}

model. A $p$ value $<0.05$ was regarded statistically significant. Statistical analysis was performed using SPSS version 19.0 for Windows (SPSS Inc., Chicago, IL, USA).

\section{Results}

3.1. Patient Characteristics. A total of 1069 patients with pathologically documented stage I GC were included in this study. The study population comprised of 795 (74.4\%) males and $274(25.6 \%)$ females. The median age was 60 years (range 18-93). Descriptive clinicopathological and detailed 


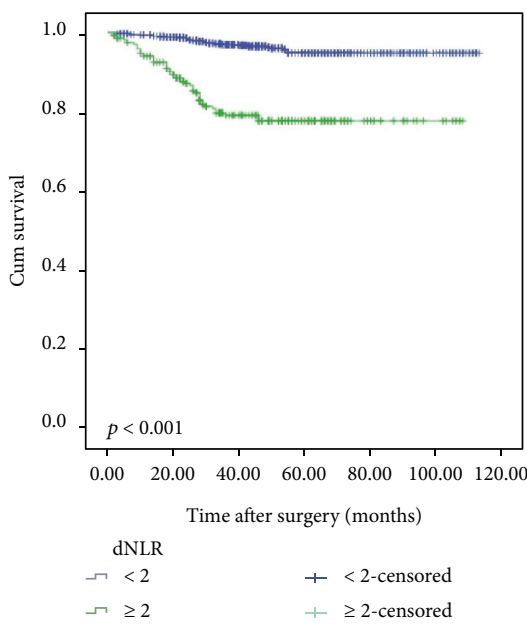

(a)

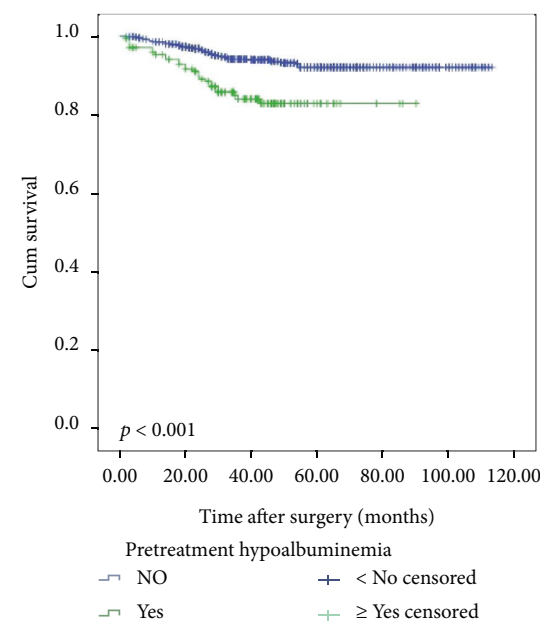

(b)

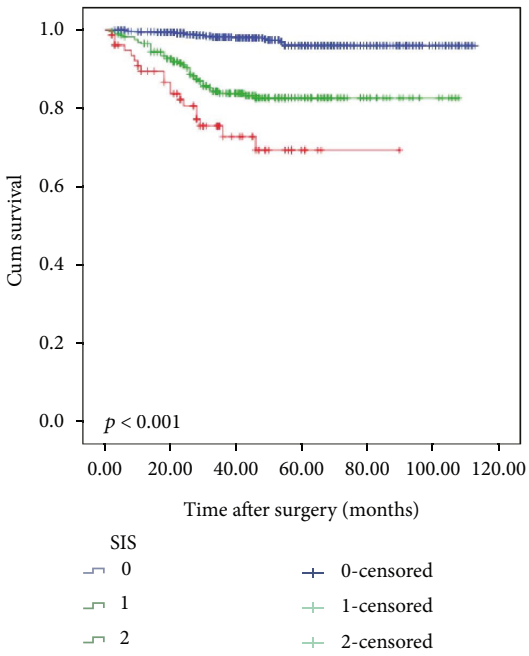

(c)

FIgURE 1: Kaplan-Meier curves for high versus low dNLR (a), with versus without pretreatment hypoalbuminemia (b), and patients according to different SIS (c) in the entire cohort.

blood count parameters of the study cohort are shown in Table 1 . The percentage of patients with $\mathrm{dNLR} \geq 2$ was $25.4 \%(272 / 1069)$ in contrast to $74.6 \%(797 / 1069)$ for those with $\mathrm{dNLR}<2$. Pretreatment hypoalbuminemia (albumin $<35 \mathrm{~g} / \mathrm{l})$ occurred in 174 patients (16.3\%). Overall, 925 (86.5\%) patients received surgery alone (the non-AC group), and 144 (13.5\%) received AC after surgery (the AC group). The baseline characteristics between the groups are shown in Table 2.

3.2. Survival. The 5-year OS rates in T1N0, T1N1, and T2N0 were $95.7 \%, 85.3 \%$, and $80.0 \%$, respectively $(p<0.001)$. There were significant differences in 5 -year OS between patients with T1N0 and T1N1 $(p<0.001)$ or T2N0 tumors $(p<0.001)$. However, no difference was observed in OS between patients with T1N1 tumors and T2N0 tumors $(p=0.461)$ (Supplementary Figure 1a). The 5-year OS was significantly higher in stage IA patients than in stage IB patients (Supplementary Figure 1b).
The 5-year OS was significantly worse in high dNLR patients than in low dNLR patients $(77.6 \%$ versus $94.7 \%$, $p<0.001$; Figure 1(a)). Patients with and without pretreatment hypoalbuminemia also differed significantly in 5-year OS $(82.9 \%$ versus $92.1 \%, p<0.001$; Figure $1(\mathrm{~b}))$.

3.3. Predictive Factors for Survival. The statistically significant prognostic factors identified by univariate analyses are shown in Table 3. Based on the multivariate analysis, the elevated pretreatment dNLR and hypoalbuminemia were independent prognostic factors for OS, together with TNM stage and lymphovascular invasion (Table 3 ).

3.4. Establishment and Prognostic Impact of the Novel Systemic Immune Score (SIS). Based on the systemic immune prognostic factors identified in the multivariate analysis, we combined the two factors to establish the SIS defined as follows: patients with both high dNLR and the pretreatment hypoalbuminemia were assigned a score of 2 , patients with either high dNLR or the pretreatment hypoalbuminemia 
TABLe 3: Univariate and multivariate Cox proportional analysis for survival.

\begin{tabular}{|c|c|c|c|c|c|}
\hline \multirow{2}{*}{ Parameter } & \multirow{2}{*}{ Categories } & \multicolumn{2}{|c|}{ Univariate analysis } & \multicolumn{2}{|c|}{ Multivariate analysis } \\
\hline & & $\operatorname{HR}(95 \% \mathrm{CI})$ & $p$ & $\mathrm{HR}(95 \% \mathrm{CI})$ & $p$ \\
\hline Age & $>65$ versus $<65$ & $1.459(0.974-2.186)$ & 0.067 & & \\
\hline Gender & Male versus female & $1.408(0.853-2.327)$ & 0.181 & & \\
\hline Tumor location & Upper versus other & $1.254(0.779-2.019)$ & 0.351 & & \\
\hline Tumor size & $\geq 30 \mathrm{~mm}$ versus $<30 \mathrm{~mm}$ & $1.555(1.041-2.324)$ & 0.031 & $1.302(0.731-1.816)$ & 0.452 \\
\hline Lymphovascular invasion & Yes versus no & $2.660(1.509-4.689)$ & 0.001 & $2.314(1.419-4.417)$ & 0.010 \\
\hline Tumor differentiation & Undifferentiated versus differentiated & $1.392(0.933-2.077)$ & 0.105 & & \\
\hline Extent of lymph node dissection & $\mathrm{D} 1+/ \mathrm{D} 1$ versus $\mathrm{D} 2$ & $1.158(0.643-1.391)$ & 0.211 & & \\
\hline AJCC TNM stage & IB versus IA & $10.183(5.765-17.987)$ & $<0.001$ & $8.098(5.201-13.145)$ & $<0.001$ \\
\hline CEA level & Above normal versus normal & $2.433(1.440-4.110)$ & 0.001 & $1.488(0.873-2.426)$ & 0.319 \\
\hline CA 19-9 level & Above normal versus normal & $1.363(0.789-2.356)$ & 0.267 & & \\
\hline Adjuvant chemotherapy & No versus yes & $1.557(0.784-3.092)$ & 0.206 & & \\
\hline dNLR & $\geq 2$ versus $<2$ & $4.357(2.910-6.524)$ & $<0.001$ & $2.464(1.301-4.312)$ & 0.004 \\
\hline Pretreatment hypoalbuminemia & Yes versus no & $2.181(1.404-3.387)$ & 0.001 & $2.102(1.254-3.576)$ & 0.028 \\
\hline SIS classification & 2 versus $\leq 1$ & $4.955(3.175-7.733)$ & $<0.001$ & $3.023(1.977-6.501)$ & 0.002 \\
\hline
\end{tabular}

CA: carbohydrate antigen; CEA: carcinoembryonic antigen; CI: confidence interval; HR: hazard ratio.

were assigned a score of 1 , and patients with both low dNLR and normal albumin were assigned a score of 0 . Namely, patients were given a total score of 0,1 , and 2 based on the sum of the points.

As mentioned above, we generate three subgroups based on the SIS. We found significant differences among the three subgroups. Thus, we combined the three subgroups to establish the SIS classification as follows: the low-risk group has no risk factor ( $\mathrm{SIS}=0$ ), the medium-risk group has 1 risk factor $(\mathrm{SIS}=1)$, and the high-risk group has two risk factors (SIS $=2$ ) (Figure 1(c)). The SIS classification also was an independent prognostic factor for OS (Table 3). Supplementary Figure 2 depicts the association between the SIS and TNM as a bubble chart.

3.5. SIS Predicts the Benefits of Adjuvant Chemotherapy. Figure 2(a) shows the survival curves for patients with and without AC. In the entire cohort, there was no significant difference in 5-year OS between the groups with and without AC (93.4\% versus $90.0 \%, p=0.109)$. This lack of a difference was also apparent when the analysis was confined to stage IA (93.7 versus $96.0 \%, p=0.946)$ and IB (83.7\% versus $79.5 \%$, $p=0.426$ ) subgroup patients (Figures $2(\mathrm{~b})$ and $2(\mathrm{c})$ ). Moreover, the survival curves (Figure 3 ) show no difference between surgery alone versus surgery $+\mathrm{AC}$ according to pretreatment dNLR and hypoalbuminemia.

Surprisingly, for our original SIS system, Kaplan-Meier curves demonstrated that high-risk patients $(\mathrm{SIS}=2)$ with AC had significantly better OS than their counterparts without AC (88.9\% versus $69.4 \%, p=0.023$ ) (Figure $4(\mathrm{a})$ ), while no significant difference was observed in the low-risk and medium-risk group $(94.4 \%$ versus $97.6 \%, p=0.695$ and $91.6 \%$ versus $81.9 \%, p=0.130$ ) (Figures $4(\mathrm{~b})$ and $4(\mathrm{c})$ ). In other words, the survival benefit of $\mathrm{AC}$ was significant for patients with SIS $=2$.

\section{Discussion}

With the increase in the detection of stage I GC, the number of stage I GC patients who experience recurrence has also increased, which may result in a large health problem in the real world $[2,8]$. It is thus important to identify patients with apparent early-stage disease who will not be cured by surgery alone and should receive adjuvant chemotherapy (AC). To date, almost all of the data on AC are derived from largescale clinical trials that included a large proportion of stages II and III patients but no or too few patients with stage I GC [3-6]. A prospective randomized clinical trial of AC versus surgery alone in stage I GC patients at high risk of recurrence or death is still ongoing (ClinicalTrials.gov identifier NCT01917552). Therefore, it will be clinically useful if a score system can be used to select candidates for AC.

A systemic inflammatory response has been determined to be an important tumor stage-independent predictor of prognosis in various malignances [31]. Pretreatment NLR values are associated with prognosis in various solid tumors, including gastric adenocarcinoma $[15,17,32]$. In 2012, Proctor et al. [20] reported the dNLR and NLR have similar prognostic value in a large cohort of unselected cancer patients. More recently, Dalpiaz [33] and his colleges found that the pretreatment dNLR was better than NLR in terms of acting as an independent prognostic factor for cancer patients. In this study, we also clearly demonstrate that dNLR was an independent predictor of long-term survival for stage I GC patients $(p<0.001)$.

In addition to pretreatment NLR, another systemic immune factor was predictive of prognosis in stage I GC in our cohort: pretreatment hypoalbuminemia. There is a growing appreciation that pretreatment hypoalbuminemia can influence the prognosis of cancer patients, possibly through 


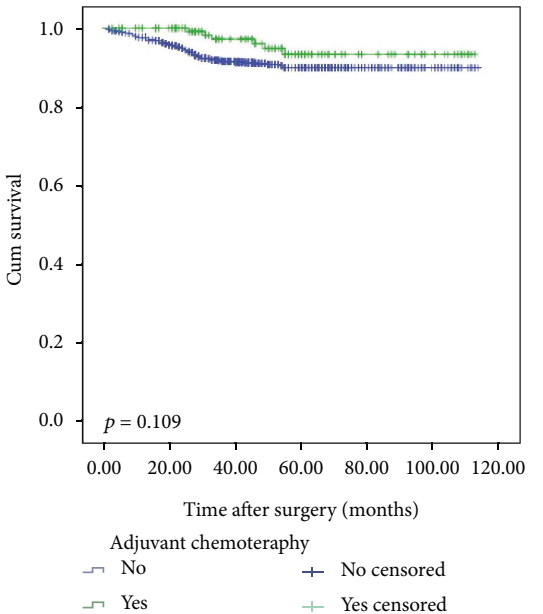

(a)

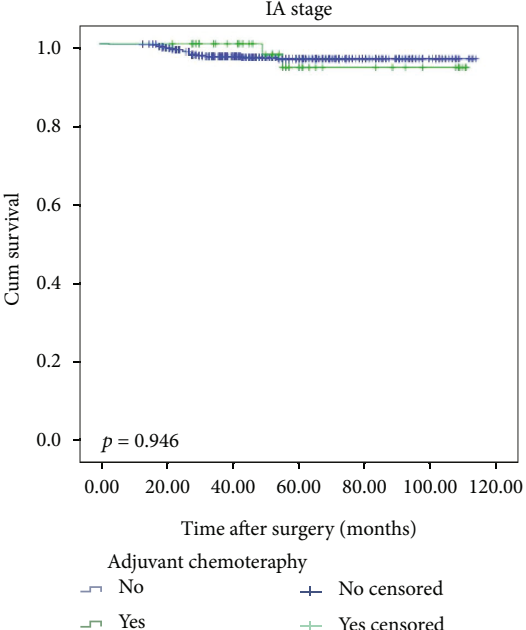

(b)

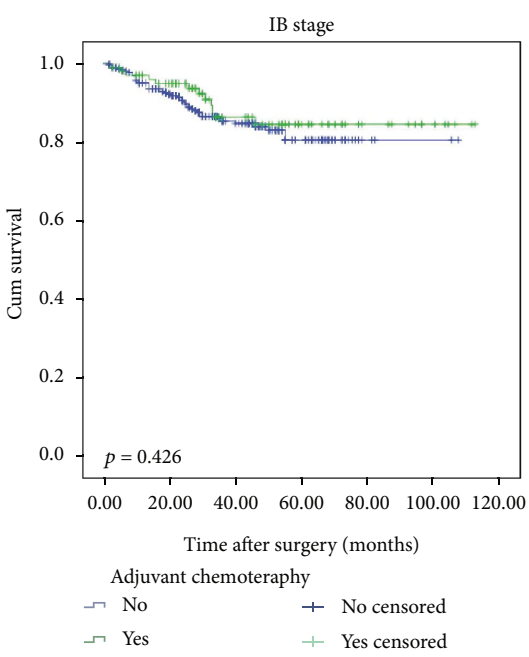

(c)

FIGURE 2: Comparison of OS between the adjuvant chemotherapy group and the surgery-only group. (a) Entire group, (b) IA stage subgroup, and (c) IB stage subgroup.

a systemic inflammatory response or impaired immunological response [34]. A recent meta-analysis of 29 studies on variety of gastrointestinal tract solid tumors reported that pretreatment serum albumin levels provide useful prognostic significance in cancer [30]. Several studies have found that low serum albumin level was an independent prognostic factor for worse outcomes in patients with gastric cancer $[35,36]$. In line with previous studies, the results of the current study show an association between pretreatment hypoalbuminemia and decreased 5-year OS.

As stated above, dNLR and hypoalbuminemia were independent prognostic predictors of survival in patients with stage I GC. Consequently, we created a novel prognostic score named the SIS based on the combination of dNLR and hypoalbuminemia, and we hypothesize that the SIS may provide further information in addition to that from classical pathological prognostic factors for identifying patients who are at high risk for poor prognosis. By grouping the patients on the basis of SIS, we identified three risk groups with distinct survival outcomes, that is, a high-risk group $(\mathrm{SIS}=2)$ with a 5-year OS rate of $71.2 \%$, a medium-risk group (SIS $=1$ ) with a 5-year OS rate of $83.1 \%$, and a low-risk group (SIS $=0$ ) with a 5 -year OS rate of $96.1 \%$. When the SIS is used for risk stratification, stage I GC patients could be categorized into three different risk groups displaying a 5-year OS rate difference of almost 25 percentage points.

Proceeding to the next step, NLR can be used to assist in risk stratification and potentially predicting immunotherapy treatment response of patients with nonmetastatic melanoma [19]. Another study from France found that pretreatment serum albumin level $>35 \mathrm{~g} / \mathrm{l}$ was the only independent predictive factor of complete response to chemoradiotherapy (CRT) in esophageal cancer patients [37]. Therefore, we hypothesized that SIS may be used to detect patients who can benefit from AC in stage I GC. For stage I gastric cancer, 


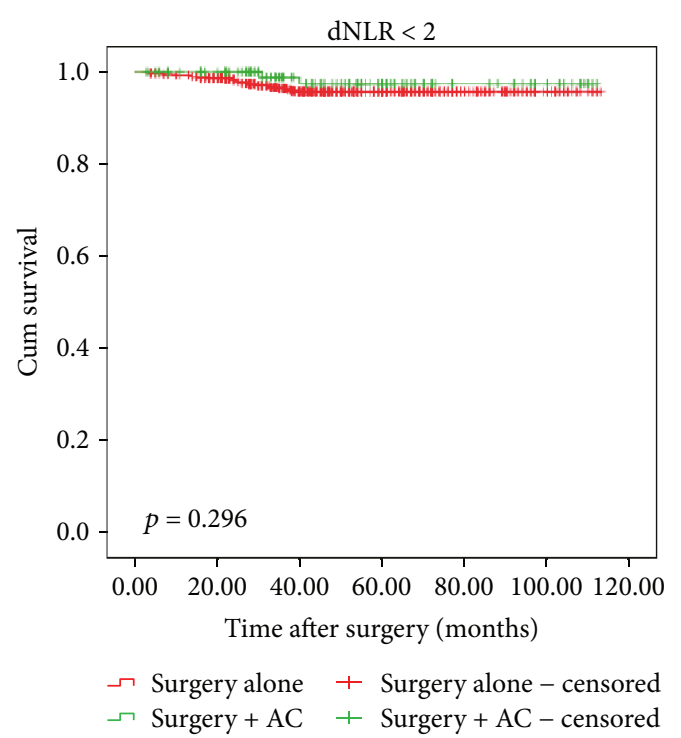

(a)

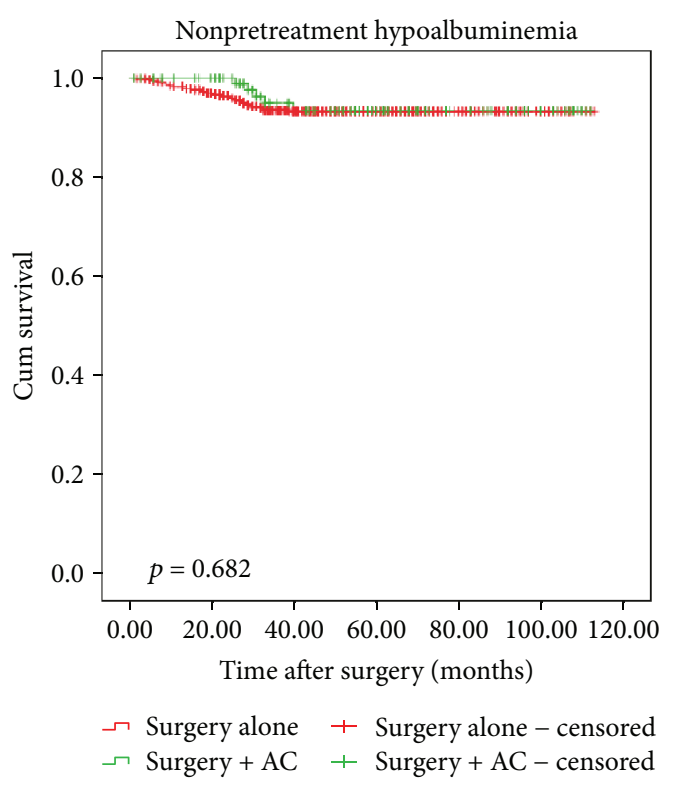

(c)

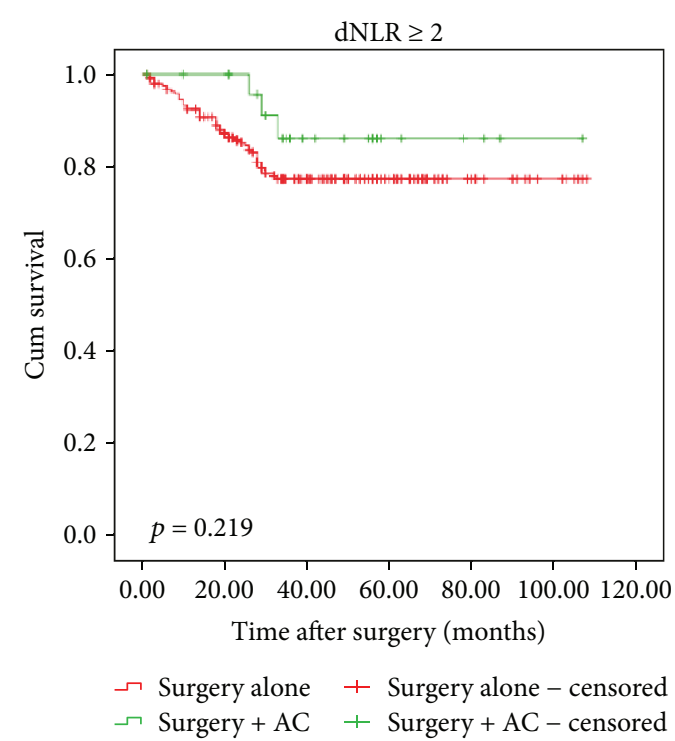

(b)

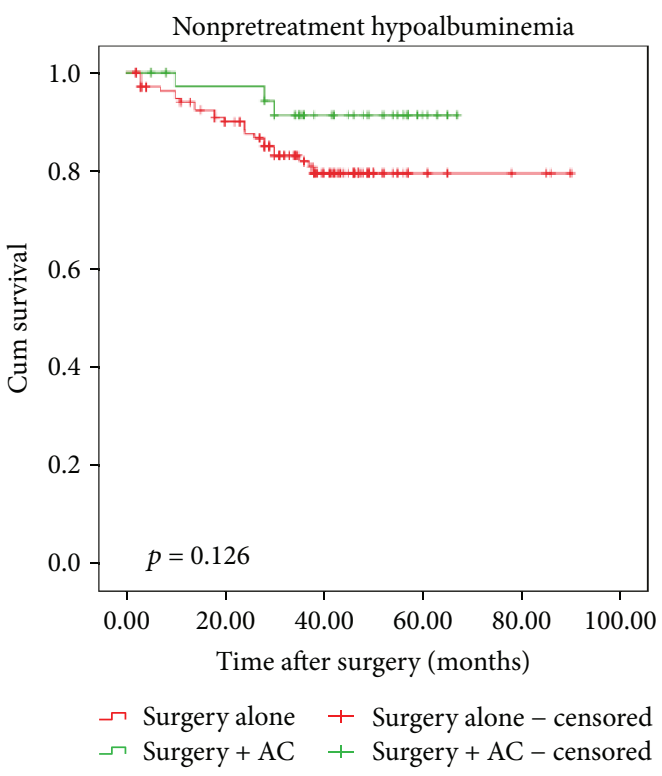

(d)

FIgURE 3: Comparison of OS between adjuvant chemotherapy group and surgery-only group. (a) dNLR $<2$ subgroup, (b) dNLR $\geq 2$ subgroup, (c) hypoalbuminemia subgroup, and (d) nonhypoalbuminemia subgroup.

poorly differentiated or higher-grade cancer, LBVI, PNI, or $<50$ years of age are considered high-risk features, and adjuvant therapy is recommended in these high-risk patients [27]. Kunisaki et al. [38] revealed that depth of invasion, lymph node metastasis, and LBVI independently influenced prognosis in stage I gastric cancer, and in their report, patients with stage I GC having lymphovascular invasion and stage II GC had similar survival outcomes; this finding suggests that adjuvant chemotherapy is suitable for stage I patients with moderate to severe lymphovascular invasion. However, none of the previous studies compared the prognosis between patients with AC and those without AC. Notably, distinct from the previous studies, we first directly compared the survival of stage I GC patients with or without AC in terms of differences in inflammation-immune status. The patients with an SIS $=2$ could achieve significantly better prognosis if treated with $\mathrm{AC}$ and should thus be considered candidates for adjuvant treatment.

There were some limitations of the present study. First, it is a single-center retrospective study. Second, we did not perform external or internal validation of our risk model. However, it might be difficult to construct a dataset including a patient population as large as ours for external validation. Despite the large cohort size in this study, the number of events was considered not insufficient to divide patients into training and validation sets for internal validation, and the 


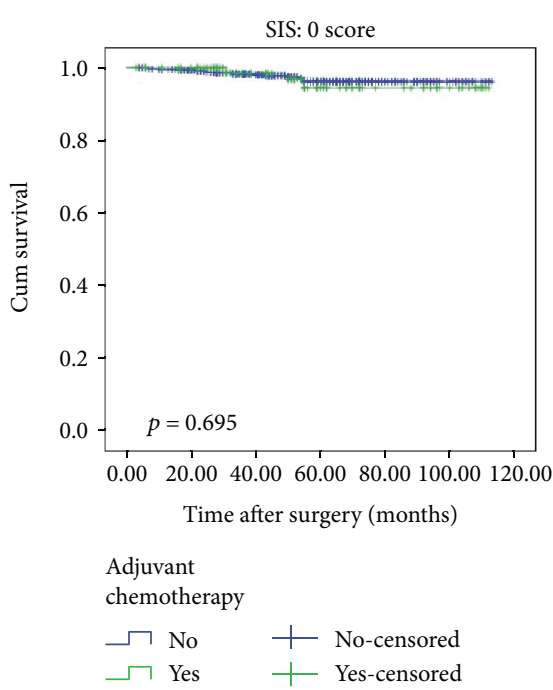

(a)

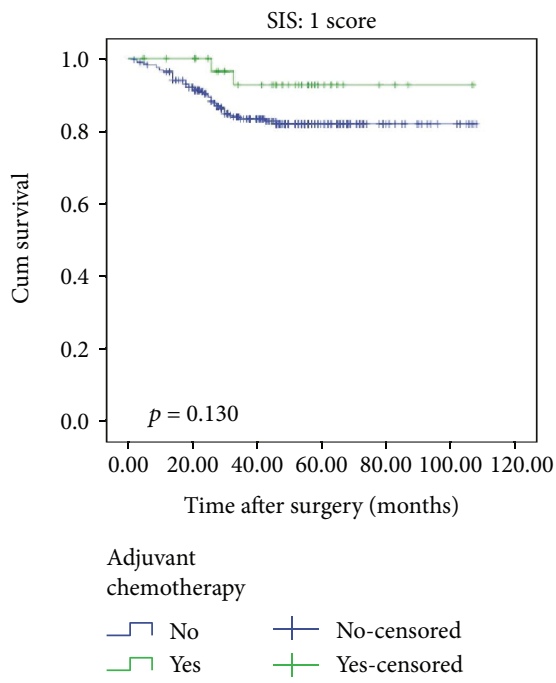

(b)

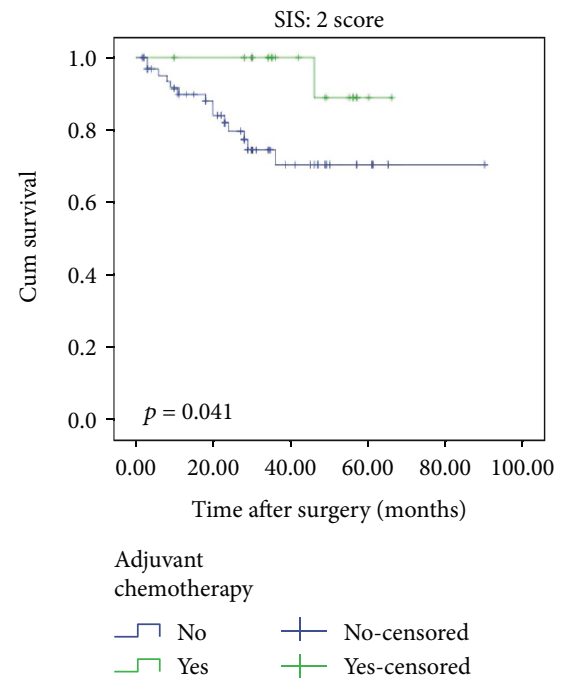

(c)

FIGURE 4: Comparison of OS between adjuvant chemotherapy group and surgery-only group. (a) 0 score subgroup, (b) 1 score subgroup, and (c) 2 score subgroup.

number of high-risk patients was relatively small. The prognostic significance of the SIS in GC patients remains to be investigated prospectively in other populations and larger cohorts in the future. Third, we did not compare the prognostic value of other SIS, such as PNI, PLR, NLR, and GPS. Finally, the optimal AC cycle for stage I patients had not yet been established.

\section{Conclusions}

The present study demonstrated that stage I GC patients with elevated pretreatment dNLR and pretreatment hypoalbuminemia were likely to receive a survival benefit from AC. In view of this, the SIS should be included in routine clinical assessments and might also be considered for risk stratification in future clinical trials of AC in early-stage GC. However, it is still necessary to conduct prospective clinical validation studies to confirm the findings of this study.

\section{Data Availability}

The data used to support the findings of this study are available from the corresponding author upon request.

\section{Ethical Approval}

All procedures performed in studies involving human participants were in accordance with the ethical standards of the institutional and/or national research committee and with the 1964 Helsinki declaration and its later amendments or comparable ethical standards.

\section{Disclosure}

The funding source hand no role in the design of this article and will not have any role during its execution or publication. 


\section{Conflicts of Interest}

The authors declare no conflict of interest.

\section{Authors' Contributions}

Jun Lu, Chao-hui Zheng, Chang-ming Huang designed this study. Jun Lu, Long-long Cao, Ping Li, Jian-wei Xie, Jia-bin Wang, and Jian-xian Lin collected the data. Jun Lu, Qi-yue Chen, Mi Lin, and Ru-hong Tu carried out the statistical analysis. Jun $\mathrm{Lu}$ and Long-long Cao drafted the manuscript. Chao-hui Zheng and Chang-ming Huang revised the manuscript. All authors read and approved the final manuscript.

\section{Acknowledgments}

This study was supported by the Scientific and Technological Innovation Joint Capital Projects of Fujian Province (2016Y9031) and the Construction Project of Fujian Province Minimally Invasive Medical Center (no. [2017]171). The second batch of special support funds for the Fujian Province Innovation and Entrepreneurship Talents (2016B013), Youth Scientific Research Subject of Fujian Provincial Health and Family Planning Commission (no. 2015-1-37), and QIHANG Funds of Fujian Medical University (no. 2016QH025).

\section{Supplementary Materials}

Supplementary 1. Table 1: characteristics of patients with stage I gastric cancer treated with gastrectomy for two treatment groups.

Supplementary 2. Figure 1: Kaplan-Meier curves for OS of stage I gastric cancer patients according to tumor stage.

Supplementary 3. Figure 2: the association between the SIS and TNM, depicted as a bubble chart.

\section{References}

[1] J. Ferlay, H. R. Shin, F. Bray, D. Forman, C. Mathers, and D. M. Parkin, "Estimates of worldwide burden of cancer in 2008: GLOBOCAN," International Journal of Cancer, vol. 127, no. 12, pp. 2893-2917, 2010.

[2] J. H. Park, M. H. Ryu, H. J. Kim et al., "Risk factors for selection of patients at high risk of recurrence or death after complete surgical resection in stage I gastric cancer," Gastric Cancer, vol. 19, no. 1, pp. 226-233, 2016.

[3] S. H. Noh, S. R. Park, H. K. Yang et al., "Adjuvant capecitabine plus oxaliplatin for gastric cancer after D2 gastrectomy (CLASSIC): 5-year follow-up of an open-label, randomised phase 3 trial," The Lancet Oncology, vol. 15, no. 12, pp. 1389-1396, 2014.

[4] S. Sakuramoto, M. Sasako, T. Yamaguchi et al., "Adjuvant chemotherapy for gastric cancer with S-1, an oral fluoropyrimidine," The New England Journal of Medicine, vol. 357, no. 18, pp. 1810-1820, 2007.

[5] M. Sasako, S. Sakuramoto, H. Katai et al., "Five-year outcomes of a randomized phase III trial comparing adjuvant chemotherapy with S-1 versus surgery alone in stage II or III gastric cancer," Journal of Clinical Oncology, vol. 29, no. 33, pp. 4387-4393, 2011.
[6] Y. J. Bang, Y. W. Kim, H. K. Yang et al., "Adjuvant capecitabine and oxaliplatin for gastric cancer after D2 gastrectomy (CLASSIC): a phase 3 open-label, randomised controlled trial," The Lancet, vol. 379, no. 9813, pp. 315-321, 2012.

[7] H. Osumi, T. Yoshio, K. Chin et al., "Chemotherapy is effective for stage I gastric cancer in patients with synchronous esophageal cancer," Gastric Cancer, vol. 19, no. 2, pp. 625-630, 2016.

[8] H. G. Youn, J. Y. An, M. G. Choi, J. H. Noh, T. S. Sohn, and S. Kim, "Recurrence after curative resection of early gastric cancer," Annals of Surgical Oncology, vol. 17, no. 2, pp. 448454, 2010.

[9] R. Cristescu, J. Lee, M. Nebozhyn et al., "Molecular analysis of gastric cancer identifies subtypes associated with distinct clinical outcomes," Nature Medicine, vol. 21, no. 5, pp. 449-456, 2015.

[10] Cancer Genome Atlas Research Network, "Comprehensive molecular characterization of gastric adenocarcinoma," Nature, vol. 513, no. 7517, pp. 202-209, 2014.

[11] J. H. Cheong, H. K. Yang, H. Kim et al., "Predictive test for chemotherapy response in resectable gastric cancer: a multicohort, retrospective analysis," The Lancet Oncology, vol. 19, no. 5, pp. 629-638, 2018.

[12] C. S. Roxburgh and D. C. McMillan, "Role of systemic inflammatory response in predicting survival in patients with primary operable cancer," Future Oncology, vol. 6, no. 1, pp. 149-163, 2010.

[13] M. Sierzega, M. Lenart, M. Rutkowska et al., "Preoperative neutrophil-lymphocyte and lymphocyte-monocyte ratios reflect immune cell population rearrangement in resectable pancreatic cancer," Annals of Surgical Oncology, vol. 24, no. 3, pp. 808-815, 2017.

[14] S. Hamada, A. Masamune, and T. Shimosegawa, "Inflammation and pancreatic cancer: disease promoter and new therapeutic target," Journal of Gastroenterology, vol. 49, no. 4, pp. 605-617, 2014.

[15] S. C. Wang, J. F. Chou, V. E. Strong, M. F. Brennan, M. Capanu, and D. G. Coit, "Pretreatment neutrophil to lymphocyte ratio independently predicts disease-specific survival in resectable gastroesophageal junction and gastric adenocarcinoma," Annals of Surgery, vol. 263, no. 2, pp. 292-297, 2016.

[16] Y. Asano, S. Kashiwagi, N. Onoda et al., "Predictive value of neutrophil/lymphocyte ratio for efficacy of preoperative chemotherapy in triple-negative breast cancer," Annals of Surgical Oncology, vol. 23, no. 4, pp. 1104-1110, 2016.

[17] G. Gemenetzis, F. Bagante, J. F. Griffin et al., "Neutrophil-tolymphocyte ratio is a predictive marker for invasive malignancy in intraductal papillary mucinous neoplasms of the pancreas," Annals of Surgery, vol. 266, no. 2, pp. 339-345, 2016.

[18] M. Chechlinska, M. Kowalewska, and R. Nowak, "Systemic inflammation as a confounding factor in cancer biomarker discovery and validation," Nature Reviews. Cancer, vol. 10, no. 1, pp. 2-3, 2010.

[19] J. L. Davis, R. C. Langan, K. S. Panageas et al., "Elevated blood neutrophil-to-lymphocyte ratio: a readily available biomarker associated with death due to disease in high risk nonmetastatic melanoma," Annals of Surgical Oncology, vol. 24, no. 7, pp. 1989-1996, 2017.

[20] M. J. Proctor, D. C. McMillan, D. S. Morrison, C. D. Fletcher, P. G. Horgan, and S. J. Clarke, "A derived neutrophil to lymphocyte ratio predicts survival in patients with cancer," British Journal of Cancer, vol. 107, no. 4, pp. 695-699, 2012. 
[21] D. C. McMillan, "The systemic inflammation-based Glasgow prognostic score: a decade of experience in patients with cancer," Cancer Treatment Reviews, vol. 39, no. 5, pp. 534-540, 2013.

[22] O. Fiala, M. Pesek, J. Finek et al., "Serum albumin is a strong predictor of survival in patients with advanced-stage nonsmall cell lung cancer treated with erlotinib," Neoplasma, vol. 63, no. 03, pp. 471-476, 2016.

[23] Japanese Gastric Cancer Association, "Japanese gastric cancer treatment guidelines 2010 (ver. 3)," Gastric Cancer, vol. 14, no. 2, pp. 113-123, 2011.

[24] K. Washington, "7th edition of the AJCC cancer staging manual: stomach," Annals of Surgical Oncology, vol. 17, no. 12, pp. 3077-3079, 2010.

[25] I. R. Cho, J. C. Park, C. H. Park et al., "Pre-treatment neutrophil to lymphocyte ratio as a prognostic marker to predict chemotherapeutic response and survival outcomes in metastatic advanced gastric cancer," Gastric Cancer, vol. 17, no. 4, pp. 703-710, 2014.

[26] H. B. Shin, J. Y. An, S. H. Lee et al., "Is adjuvant chemotherapy necessary in pT1N1 gastric cancer?," BMC Cancer, vol. 17, no. 1, p. 287, 2017.

[27] D. C1, Y. Zhou, H. Cai, G. Zhao, H. Fu, and Y. Q. Shi, "Poor prognostic factors in patients with stage I gastric cancer according to the seventh edition TNM classification: a comparative analysis of three subgroups," Journal of Surgical Oncology, vol. 105, no. 3, pp. 323-328, 2012.

[28] S. G. Barreto and J. A. Windsor, "Redefining early gastric cancer," Surgical Endoscopy, vol. 30, no. 1, pp. 24-37, 2016.

[29] H. Zhang, H. Liu, Z. Shen et al., "Tumor-infiltrating neutrophils is prognostic and predictive for postoperative adjuvant chemotherapy benefit in patients with gastric cancer," Annals of Surgery, vol. 267, no. 2, pp. 311-318, 2016.

[30] D. Gupta and C. G. Lis, "Pretreatment serum albumin as a predictor of cancer survival: a systematic review of the epidemiological literature," Nutrition Journal, vol. 22, no. 9, p. 69, 2010.

[31] S. Ghuman, M. Van Hemelrijck, H. Garmo et al., "Serum inflammatory markers and colorectal cancer risk and survival," British Journal of Cancer, vol. 116, no. 10, pp. 13581365, 2017.

[32] B. Bhindi, T. Hermanns, Y. Wei et al., "Identification of the best complete blood count-based predictors for bladder cancer outcomes in patients undergoing radical cystectomy," British Journal of Cancer, vol. 114, no. 2, pp. 207-212, 2016.

[33] O. Dalpiaz, T. Luef, M. Seles et al., "Critical evaluation of the potential prognostic value of the pretreatment-derived neutrophil-lymphocyte ratio under consideration of C-reactive protein levels in clear cell renal cell carcinoma," British Journal of Cancer, vol. 116, no. 1, pp. 85-90, 2017.

[34] D. C. McMillan, "Systemic inflammation, nutritional status and survival in patients with cancer," Current Opinion in Clinical Nutrition and Metabolic Care, vol. 12, no. 3, pp. 223-226, 2009.

[35] H. Sun, B. He, Z. Nie et al., "A nomogram based on serum bilirubin and albumin levels predicts survival in gastric cancer patients," Oncotarget, vol. 8, no. 25, pp. 41305-41318, 2017.

[36] L. F. Oñate-Ocaña, V. Aiello-Crocifoglio, D. Gallardo-Rincón et al., "Serum albumin as a significant prognostic factor for patients with gastric carcinoma," Annals of Surgical Oncology, vol. 14, no. 2, pp. 381-389, 2007.
[37] F. Di Fiore, S. Lecleire, D. Pop et al., "Baseline nutritional status is predictive of response to treatment and survival in patients treated by definitive chemoradiotherapy for a locally advanced esophageal cancer," The American Journal of Gastroenterology, vol. 102, no. 11, pp. 2557-2563, 2007.

[38] C. Kunisaki, H. Makino, J. Kimura et al., "Impact of lymphovascular invasion in patients with stage I gastric cancer," Surgery, vol. 147, no. 2, pp. 204-211, 2010. 


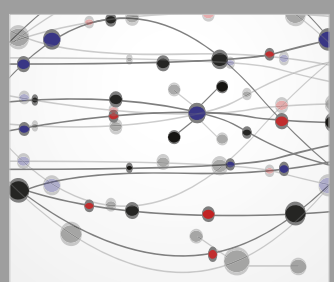

The Scientific World Journal
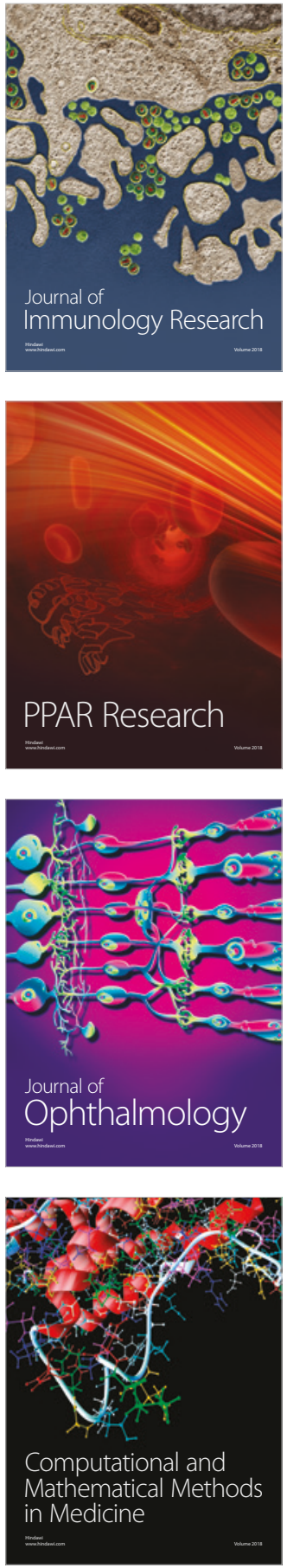

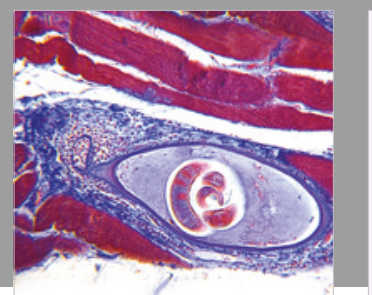

Gastroenterology Research and Practice

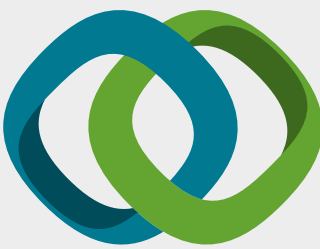

\section{Hindawi}

Submit your manuscripts at

www.hindawi.com
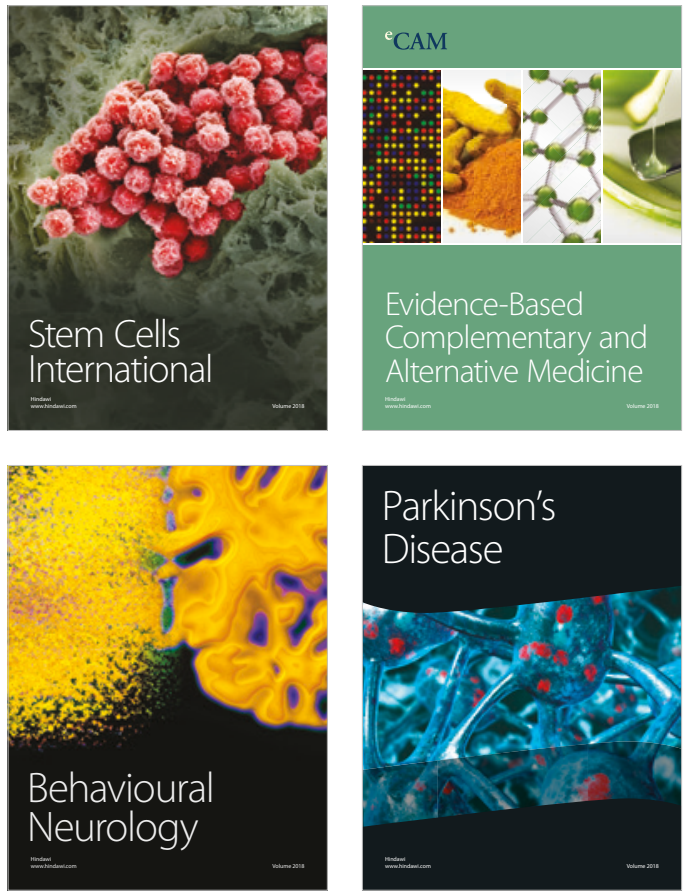

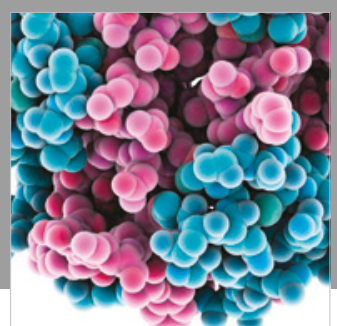

ournal of

Diabetes Research

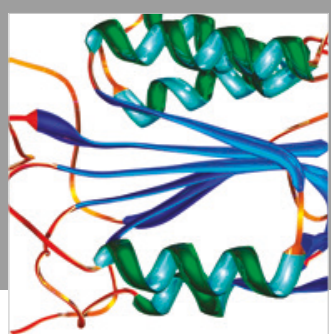

Disease Markers
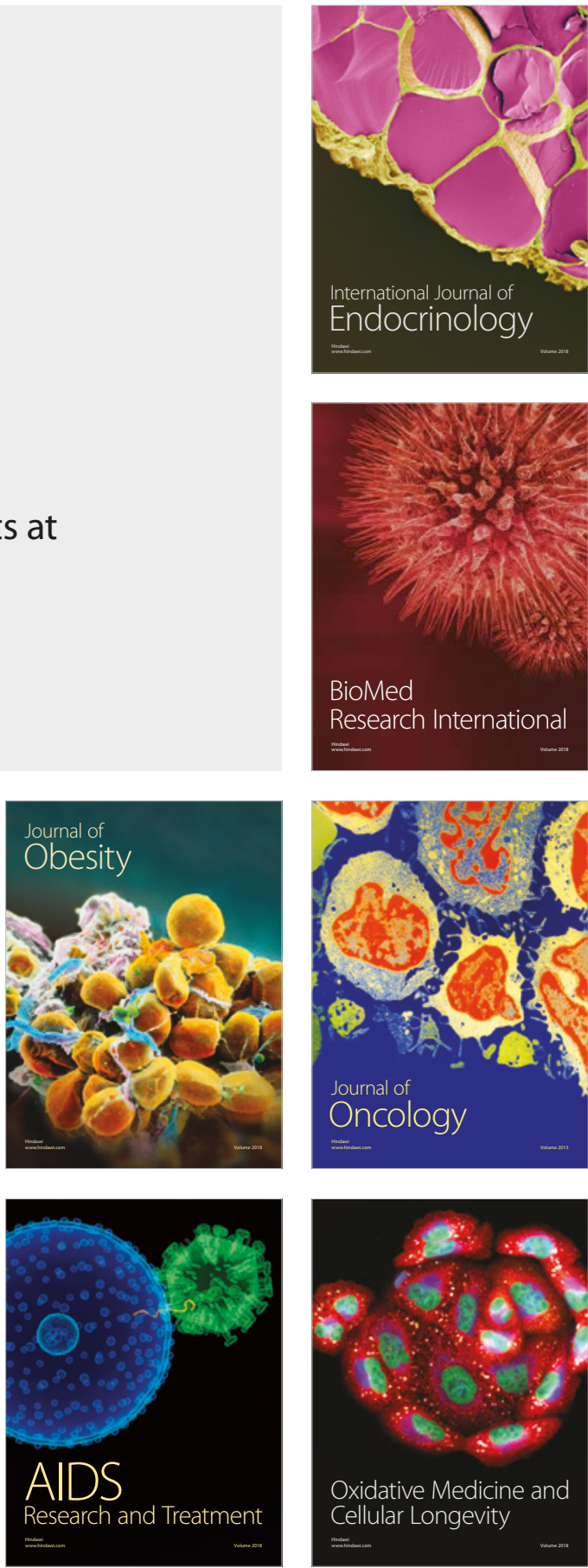